\title{
Artesunate plus sulfadoxine-pyrimethamine for treatment of uncomplicated Plasmodium falciparum malaria in Sudan Sakina B Elamin ${ }^{1}$, Elfatih M Malik ${ }^{1}$, Tarig Abdelgadir ${ }^{1}$, Ammar H Khamiss ${ }^{2}$, Mamoun M Mohammed ${ }^{3}$, Elderderi S Ahmed ${ }^{1}$ and Ishag Adam*4
}

\author{
Address: ${ }^{1}$ NationalMalaria Control, Ministry of Health, Khartoum, Sudan, ${ }^{2}$ Albayan College for Science, Sudan University for Science and \\ Technology, Sudan, ${ }^{3}$ University of Kassala, Sudan and ${ }^{4}$ Faculty of Medicine University of Khartoum, The Academy of Medical Sciences and \\ Technology, Department of Obstetrics \& Gynecology, Faculty of Medicine University of Khartoum, P. O. Box 102, Khartoum, Sudan \\ Email: Sakina B Elamin - sakinaba@hotmail.com; Elfatih M Malik - fatihmmalik@hotmail.com; \\ Tarig Abdelgadir - tarigmohamad@hotmail.com; Ammar H Khamiss - Ammmarhasssan@ hotmail.com; \\ Mamoun M Mohammed - mosmanmm@hotmail.com; Elderderi S Ahmed - dirsalim2@yahoo.com; Ishag Adam* - ishagadamm@yahoo.com \\ * Corresponding author
}

Published: 14 September 2005

Malaria Journal 2005, 4:4I doi:|0.1|186/|475-2875-4-4|

This article is available from: http://www.malariajournal.com/content/4/I/4 I

(C) 2005 Elamin et al; licensee BioMed Central Ltd.

This is an Open Access article distributed under the terms of the Creative Commons Attribution License (http://creativecommons.org/licenses/by/2.0), which permits unrestricted use, distribution, and reproduction in any medium, provided the original work is properly cited.
Received: 19 June 2005

Accepted: 14 September 2005

\begin{abstract}
Background: Early diagnosis and effective treatment with an appropriate drug form the main components of the World Health Organization's strategy to reduce malaria related mortality. The few available drugs might be safeguarded if combined with artesunate. The addition of artesunate to a standard antimalarial treatment substantially reduces treatment failure, recrudescence and gametocyte carriage.
\end{abstract}

Methods: During late 2004, the efficacy of artesunate $(4 \mathrm{mg} / \mathrm{kg}$. day, on days $0-2)$ plus sulfadoxinepyrimethamine $(25 \mathrm{mg} / \mathrm{kg}$, on day 0$)$ for the treatment of uncomplicated Plasmodium falciparum malaria was investigated in four sentinel areas in Sudan, with different malaria transmission (Damazin, Kassala, Kosti, and Malakal).

Results: Two hundreds and sixty-nine patients completed the 28-day follow-up. On day one, 60 (22.3\%) patients were febrile and $15(5.5 \%)$ patients were parasitaemic. On day three, all the patients were afebrile and aparasitaemic. While two patients $(0.7 \%$, Kassala) showed late Clinical and Parasitological Failures, the rest $(99.3 \%)$ of the patients demonstrated Adequate Clinical and Parasitological Response. A gametocytaemia were detected during the follow-up in one patient (0.37\%, Kassala). Adverse drug effects were detected in 32 (1 I.9\%) patients

Conclusion: The study showed that AS plus SP is an effective, safe drug in the treatment of uncomplicated $P$. falciparum malaria in Sudan.

\section{Background}

There are almost 515 (range 300-660) million episodes of clinical Plasmodium falciparum malaria infections [1]. Drug-resistant malaria is spreading in Africa and countries with high levels of resistance have witnessed increased morbidity and mortality [2]. Early diagnosis and effective treatment with an appropriate drug form the main components of the World Health Organization's strategy to reduce malaria-related mortality [3]. 
The few available drugs might be safeguarded if combined with artesunate. The addition of artesunate to standard antimalarial treatments substantially reduces treatment failure, recrudescence and gametocyte carriage, preventing the emergence and spread of drug resistance and interrupting the transmission of $P$. falciparum. Coupled with early detection and confirmed diagnosis, this strategy represents the only way forward in the chemotherapy of malaria [4-8].

Malaria causes between 7.5 to 10 million cases and 35,000 deaths every year in Sudan [9]. Due to the spread of multidrug-resistant $P$. falciparum malaria in Sudan $[10,11]$, artesunate plus sulfadoxine-pyrimethamine is recommended as the first-line treatment for uncomplicated $P$. falciparum malaria. The study aimed to investigate the efficacy of AS plus SP, as there is little published data in Sudan $[8,12]$.

\section{Patients and methods \\ Data collection}

The study was conducted in October and November, 2004 at four health centres in different regions of Sudan (Damazin, Kassala, Kosti, and Malakal) (Figure 1). Three of these areas were characterized by low malaria transmission and the fourth (Malakal) was characterized by stable transmission [13]. Febrile (temperature $\geq 37.5^{\circ} \mathrm{C}$ ) patients with uncomplicated $P$. falciparum malaria [14], who had no history of antimalarial drug use during the preceding two weeks, were recruited for the study. Pregnant women and patients with mixed infections were excluded.

After obtaining informed consent from the patient or the child's parents, a fixed questionnaire including relevant socio-demographic characteristics, medical history, physical findings and investigations conducted was completed for each patient.

\section{Laboratory methods}

Blood films were prepared, stained with Giemsa and 100x oil immersion fields were examined. The parasite density was counted against 200 leucocytes, assuming 6,000 leucocytes $/ \mu \mathrm{l}$. All the slides were double-checked blindly and only considered negative if no parasites were detected in 100 oil immersion fields. If gametocytes were seen, then the count was extended to 500 leucocytes.

\section{Treatment and follow up}

The patients were given the AS plus SP combination, with artesunate ( $4 \mathrm{mg} / \mathrm{kg}$. day) given on days $0-2$ and a single dose of SP (25 mg/kg) (Dafra Pharma, Beerse, Belgium) given on day 0 . The tablets were crushed and dissolved in water for children who were not able to swallow them. Subjects were observed for vomiting for one hour; the full

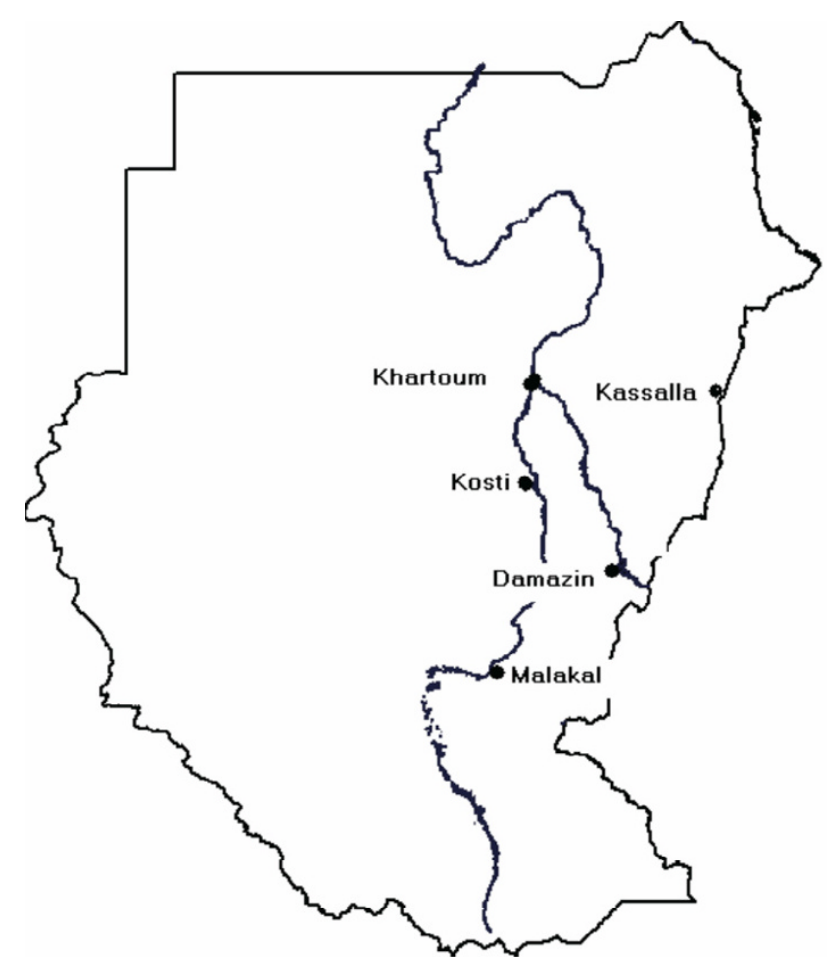

Figure I

A sketch map of the Sudan, showing the main rivers, Khartoum and the four sites where the study was conducted.

dose was repeated for those who vomited within $30 \mathrm{~min}$ and half of the dose was repeated if vomiting occurred between 30 and 60 minutes.

\section{Follow-up and re-treatment}

Patients were requested to come on days 1, 2, 3, 7, 14, 21 and 28 and at any time if they felt unwell. At each visit, body temperature was measured and blood films were prepared. During the follow-up the patients were asked if they suffered from side effects which can be expected from antimalarial treatment (nausea, vomiting, abdominal pain, dizziness and rash); these symptoms were considered to be drug related if they had not been reported at the patient's first presentation in the clinic.

Quinine was given for treatment failures. Early Treatment Failures (ETF) in case of significant parasitaemia at day 2 or 3 or parasites and fever at day 3. Late Clinical Failures (LCF) for cases with parasites and fever during follow-up after day 3 and Late Parasitological Failures (LPF) for parasite infections with/without fever during the follow-up. Cases which remained negative during follow-up were considered Adequate Clinical and Parasitological 
Table I: The base line (day 0) characteristics of the 269 patients who completed the 28 days of follow-up after the treatment with artesunate plus sulfadoxine-pyrimethamine*.

\begin{tabular}{lllllll}
\hline Variable & Total $(N=269)$ & Damazin $(N=72)$ & Kassala $(N=50)$ & Kosti $(N=70)$ & Malakal $(N=77)$ & Significance \\
\hline Age, years & $12.1(12.4)$ & $10.6(9.0)$ & $24.3(15.4)$ & $14.7(12.08)$ & $3.4(1.2)$ & $\mathrm{P}<0.05$ \\
Weight, $\mathrm{Kg}$ & $28.1(19.3)$ & $25.6(14.5)$ & $47.4(21.5)$ & $33.2(18.5)$ & $3.2(0.36)$ & $\mathrm{P}<0.05$ \\
Temperature, ${ }^{\circ} \mathrm{C}$ & $38.2(0.76)$ & $38.1(0.6)$ & $38.2(0.6)$ & $37.9(0.5)$ & $38.6(0.9)$ & $\mathrm{P}>0.05$ \\
Parasite count, rings $/ \mu$ & $25532.3(24196.2)$ & $21924.3(17748.7)$ & $32240.3(28372.9)$ & $11509.8(11135.2)$ & $37297.5(27844.5)$ & $\mathrm{P}<0.05$ \\
Children $<5$ years & $100(37.0)$ & $12(16.7)$ & $5(10)$ & $12(17.1)$ & $71(92.2)$ & $\mathrm{P}<0.05$ \\
Female & $127(47.2)$ & $37(51.4)$ & $18(36)$ & $32(45.7)$ & $40(51.9)$ & $\mathrm{P}>0.05$ \\
\hline
\end{tabular}

*Data were shown as mean (SD) or numbers (\%) as appropriate

Responses (ACPR). These were modified WHO guidelines $[14,15]$.

\section{Statistics}

Data were entered into a computer database and SPSS software (SPSS Inc., Chicago, IL, USA) was used for statistical analysis. The means (age, weight, temperature and parasite count) were calculated for all the patients and were compared between the patients in the different locations using one way analysis of the variance (ANOVA), when the data is normally distributed and by the Kruskal Wallis test if the data was not normally distributed. Percentages were calculated and compared for the patients in the four locations by an $\chi^{2}$ test. $P<0.05$ was regarded significant.

\section{Ethical clearance}

The study received ethical clearance from the Sudanese National Malaria Administration.

\section{Results}

Two hundred and ninety $(32.5 \%)$ out of 890 screened patients fulfilled the criteria and were enrolled in the study. Twenty-one (7.2\%) of these were lost in the followup and 269 patients $(72,50,70$, and 77 from Damazin, Kassala, Kosti, and Malakal, respectively) completed the 28-day follow-up. Their different characteristics are shown in Table 1 . The mean age and weight were significantly higher in the Kassala group. The parasite count was significantly higher in the Malakal area. 37.0\% (100 patients) were children less than five years old; this proportion was significantly higher in Malakal group $(92.2 \%$, see Table 1). One hundred and twenty seven subjects $(47.2 \%)$ were females; their percentages were not significantly different within the groups (see Table 1).

On day one, $60(22.3 \%)$ patients were febrile and 15 $(5.5 \%)$ patients were parasitaemic. By day three all the patients were afebrile and aparasitaemic. There were two (0.7\%) Late Clinical and Parasitological Failures (days 7 and 22) from Kassala, there was no Clinical and Parasito- logical Failures from other locations (Table 2). Only one patient (Kassala) showed gametocytaemia on day 14 of the follow-up. Thirty two (11.9\%) patients suffered expected adverse effects (nausea, itching and dizziness), but these were mild and resolved spontaneously.

\section{Discussion}

The study investigated the efficacy of AS plus SP for the treatment of uncomplicated $P$. falciparum malaria at four sites in the Sudan. This is probably the largest study reporting AS plus SP efficacy in Sudan until now. Although, the baseline characteristics (age and parasite count) were significantly different between the four locations, the study showed that two (0.7\%, Kassala) out of 269 patients were found to have Late Clinical and Parasitological Failures. Since the parasite genotyping (PCR) was not conducted, the possibility of re-infection/recrudescence is still there. Hundred percent efficacy of AS plus SP was recently reported from eastern Sudan [8] and 99\% from southern Sudan [12]. The high cure rate in this study is comparable to that reported from neighbouring African countries $[5,6]$. However, the highest drug resistant $P$. falciparum strains were reported from eastern Sudan $[11,16]$. The expected adverse effects (nausea, itching and dizziness) were reported in $(11.9 \%)$ of the patients in this study. These results were in line with, observations of others, where the adverse effects were not significantly different, if compared with those of SP alone [5,8].

The adverse effects (nausea, vomiting) might influence the adherence to AS plus SP, especially science this therapy is only available in the oral form, which is not the medication preferred by Sudanese patients [17]. Furthermore, adherence may be influenced by the multiple doses of the combination, rather the previous single dose of SP, which was reported to be the most important single factor for the best adherence of SP among Sudanese patients [17].

A post- treatment gametocytaemia was detected in one patient in Kassala area. High (20\%) levels of gametocytae- 
Table 2: Trail profile, showing number of patients enrolled, treated and completing the 28 days of follow-up after the treatment with artesunate plus sulfadoxine-pyrimethamine*.

\begin{tabular}{|c|c|c|c|c|c|}
\hline Variable & Total & Damazin & Kassala & Kosti & Malakal \\
\hline The recruited patients & 290 & 77 & 53 & 76 & 84 \\
\hline Lost to follow-up & $21(7.2)$ & $5(6.5)$ & $3(5.6)$ & $6(7.9)$ & $7(8.3)$ \\
\hline Patients completed the 28-days of follow-up & $269(94.8)$ & $72(93.5)$ & $50(94.4)$ & $70(92.1)$ & 77 (9।.7) \\
\hline Accurate clinical and parasitological response & $267(99.3)$ & $72(100)$ & $48(96)$ & $70(100)$ & $77(100)$ \\
\hline Late clinical and parasitological failure & $2(0.7)$ & $0(0)$ & $2(4)$ & $0(0)$ & $0(0)$ \\
\hline
\end{tabular}

*Data were shown as numbers (\%).

mia had been reported in the eastern Sudan following SP, quinine and mefloquine treatment [18-20]. However, it has not been reported during the follow-up of patients in the eastern Sudan treated with artemether, artesunate plus mefloquine or AS plus SP $[8,19,21]$. The ability of artesunate to reduce the post-treatment gametocytaemia is important, as it may reduce transmission [4].

\section{Conclusion}

The study showed that, As plus SP is an effective, safe drug in the treatment of uncomplicated $P$. falciparum malaria in Sudan.

\section{Authors' contributions}

SBE, EMM, TA, MTM, ESA carried out the study in the different sites and participated in the statistical analysis and procedures, AHK participated in the statistical analysis, IA coordinated and participated in the design of the study, statistical analysis and the drafting of the manuscript. All the authors read and approved the final version.

\section{References}

I. Snow RW, Guerra CA, Noor AM, Myint HY, Hay SI: The global distribution of clinical episodes of Plasmodium falciparum malaria. Nature 2005, 434:214-217.

2. Zucker JR, Ruebush TK, Obonyo C, Otieno J, Campbell CC: The mortality consequences of the continued use of chloroquine in Africa: experience in Siaya, western Kenya. Am J Trop Med Hyg 2003, 68:386-390.

3. World Health Organization: A global strategy for malaria control. Geneva WHO; 1993.

4. Nosten F, Brasseur P: Combination therapy for malaria: the way forward? Drugs 2002, 62:1315-1329.

5. Obonyo CO, Ochieng F, Taylor WR, Ochola SA, Mugitu K, Olliaro P, ter Kuile $\mathrm{F}$, Oloo Al: Artesunate plus sulfadoxine-pyrimethamine for uncomplicated malaria in Kenyan children: a randomized, double-blind, placebo-controlled trial. Trans $R$ Soc Trop Med Hyg 2003, 97:585-59I.

6. Priotto G, Kabakyenga J, Pinoges L, Ruiz A, Eriksson T, Coussement F, Ngambe T, Taylor WR, Perea W, Guthmann JP, Olliaro P, Legros $D$ : Artesunate and sulfadoxine-pyrimethamine combinations for the treatment of uncomplicated Plasmodium falciparum malaria in Uganda: a randomized, double-blind, placebocontrolled trial. Trans R Soc Trop Med Hyg 2003, 97:325-330.

7. Adjuik M, Babiker A, Garner P, Olliaro P, Taylor W, White N: Artesunate combinations for treatment of malaria: metaanalysis. Lancet 2004, 363:9-I7.

8. Adam I, A/Elbasit I, Idris SM, Malik EM, Elbashir MI: A comparison of the efficacies of artesunate plus sulfadoxine-pyrimeth- amine versus sulfadoxine-pyrimethamine alone, in the treatment of uncomplicated Plasmodium falciparum malaria in eastern Sudan. Ann Trop Med Parasitol 2005:449-455.

9. Malik EM, Khalafalla OM: Malaria in Sudan: past, present and the future. Gezira Journal of Health Sciences 2004, I (suppl):47-5 I.

10. Adam I, Osman ME, ElGhazali G, Ahmed GI, Gustafson LL, Elbashir MI: Efficacies of chloroquine, sulphadoxine-pyrimethamine and quinine for the treatment of uncomplicated Plasmodium falciparum malaria in Eastern Sudan. Ann Trop Med Parasitol 2004, 98:66I-666.

II. Adam I, Elbashir MI: Resistance of $\boldsymbol{P}$. falciparum to antimalarial drugs in Sudan: Review. Khartoum Med J 2005 in press.

12. van den Broek I, Amsalu R, Balasegaram M, Hepple P, Alemu E, Hussein B, Al-Faith M, Montgomery J, Checchi F: Efficacy of two artemisinin combination therapies for uncomplicated falciparum malaria in children under 5 years, Malakal, Upper Nile, Sudan. Malar J 2005, 4: I4.

13. Al Gadal AA: Malaria in the Sudan. In Proceedings of the Conference on Malaria in Africa Edited by: Buck AA. Washington, DC: American Institute of Biological Sciences; 1986:156-159.

14. World Health Organization: Assessment of therapeutic efficacy of antimalarial drugs for uncomplicated falciparum malaria in areas with intense transmission. In Document WHOIMAL/ 96.1077 Geneva: WHO; 1996.

15. WHO: Assessment and Monitoring of Antimalarial Drug Efficacy for the treatment of uncomplicated Falciparum malaria. WHO/HTM/RBM/2003.50 2003.

16. Salah MT, Mohammed MM, Himeidan YE, Malik EM, Elbashir MI, Adam I: A randomized comparison of sulphadoxine-pyrimethamine and combination of sulphadoxine-pyrimethamine with chloroquine in the treatment of uncomplicated falciparum malaria in Eastern Sudan. Saudi Med J 2005, 26: I 47- I 48.

17. Abdel-Humeed AA: Malaria case management at the community level in Gezira Sudan. Afr J Med Sc 200I, 30:43-46.

18. Adam I, Ibrahim MH, A/Elbasit I, Elbashir MI: Efficacy of sulphadoxine-pyrimethamine in the treatment of uncomplicated Plasmodium falciparum malaria in a small sample of Sudanese children. East Mediterr Health $\mathrm{f} 2004$ in press.

19. Adam I, A/Elbasit I, Elbashir MI: Efficacies of mefloquine alone and artesunate followed by mefloquine for the treatment of uncomplicated Plasmodium falciparum malaria in eastern Sudan. Ann Trop Med Parasitol 2005, 99: I I I-I I 7.

20. Adam I, Idris H, Mohamed-Ali AA, A/Elbasit IA, Elbashir MI: Quinine versus artemether in the treatment of severe falciparum malaria in Sudanese children. East Afr Med J 2002, 79:62I-625.

21. Adam I, Salih I, Elbashir MI: Quinine for the treatment of uncomplicated Plasmodium falciparum malaria in eastern Sudan. Trans R Soc Trop Med Hyg 2005, 90:736-738. 